\title{
Intracerebral hemorrhage due to moyamoya syndrome as a rare presentation of cerebral rheumatoid vasculitis: a case report
}

\author{
Ayman Hassan El-Sudany ${ }^{1 *}$, Salah Hussein El-Halawany ${ }^{2}$, Shady S. Georgy ${ }^{1}$, Amr S. Zaki ${ }^{1}$, Rady Y. Bedros ${ }^{1}$, \\ Mostafa Hashim Mostafa ${ }^{1}$ and Ahmed El-Bassiouny ${ }^{1}$
}

\begin{abstract}
Background: Central nervous system affection in rheumatoid arthritis is rare. The most frequently encountered neurological complications with rheumatoid arthritis are peripheral neuropathy and atlantoaxial subluxation with subsequent spinal cord compression. Cerebral rheumatoid vasculitis is not a common complication.
\end{abstract}

Case presentation: A 60-year-old Egyptian female with history of rheumatoid arthritis for 15 years presented with headache and receptive aphasia. Computed tomography scan of the brain showed recent intracerebral hemorrhage. Digital subtraction cerebral angiography showed moyamoya syndrome angiographic pattern as sequelae of intracranial vasculitis. The patient did well with conservative management.

Conclusion: Cerebral rheumatoid vasculitis is rare, but can be a life-threatening condition. Early management of rheumatoid arthritis is essential to prevent such serious complication.

Keywords: Rheumatoid arthritis, Cerebral rheumatoid vasculitis, Intracerebral hemorrhage, Moyamoya syndrome, Digital subtraction angiography

\section{Background}

Rheumatoid arthritis (RA) is a chronic systemic inflammatory autoimmune disease which primarily affects the small joints of the hands and feet. Rheumatoid vasculitis (RV) typically happens with long-standing RA or with high levels of rheumatoid factor (RF) and anti-cyclic citrullinated peptide (anti-CCP) [1]. RV occurs due to immune-complex deposition within the sub-endothelium of affected vessels, a mechanism that will be increased in patients with high levels of RF and anti-CCP [2]. However, central nervous system vasculitis in RA is sort of rare and the literature contains solely few case reports on intracranial arteritis presenting as intracerebral hemorrhage (ICH). [3].

\footnotetext{
*Correspondence: aymanhsudany@gmail.com

${ }^{1}$ Neurology Department, Faculty of Medicine, Ain Shams University,

Cairo, Egypt

Full list of author information is available at the end of the article
}

\section{Case presentation}

A 60-year-old Egyptian female with irrelevant family history, past history of RA of 15 years duration, mainly in the form of small joint arthritis, otherwise no relevant past history, presented to the emergency room with sudden onset of persistent headache, inability to understand speech with in appropriate response and poor communication with her family. On examination, her vital signs were within the normal range. Neurological evaluation revealed that the patient suffers from receptive aphasia which is the cause of her symptoms. The rest of the neurological examination was normal. Computed tomography (CT) of the brain (General Electric, United States) showed left temporoparietal hematoma as shown in Fig. 1. Fundus examination showed no papilledema.

After discussion with the attending neurosurgeon, the patient was candidate for conservative management. She was admitted to the intensive care unit (ICU). The 

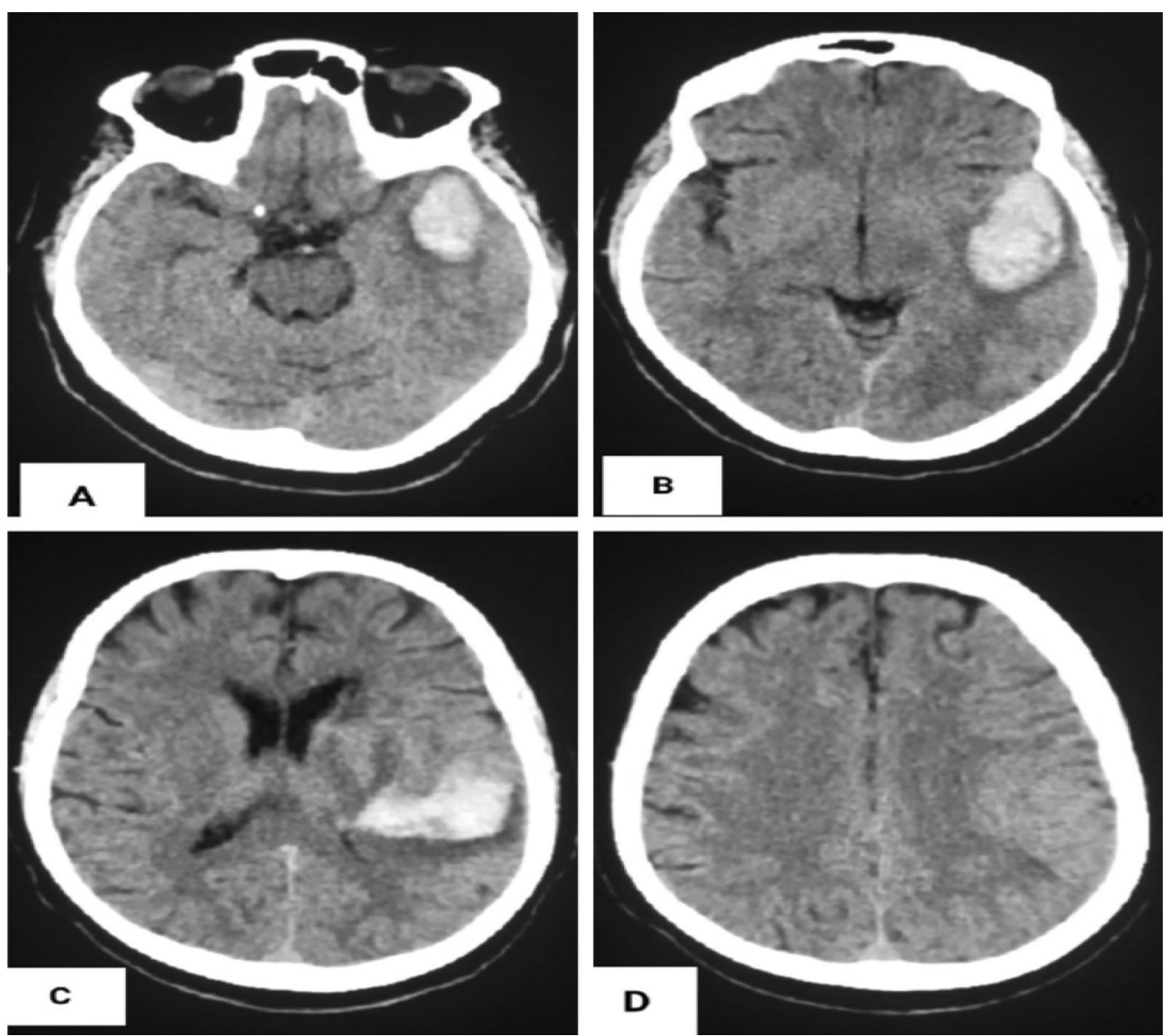

Fig. 1 A-D Computed tomography scan of the brain showing left temporoparietal hemorrhage

patient received intravenous mannitol 20\% (100 ml/6 h. for 5 days) as a brain dehydrating measure and neuroprotective therapy. Serial CT brain showed stationary size of the hemorrhage. After $48 \mathrm{~h}$ of ICU monitoring, the patient was stable and no further neurological deficit developed, so she was transferred to the medical ward.

Due to the unusual location of the intracranial hematoma, digital subtraction angiography (DSA) was requested. The procedure was done using a monoplane neurovascular machine (Siemens, Germany). DSA revealed occlusion of the distal parts of both internal carotid arteries with filling of both middle cerebral arteries through a newly formed network of blood vessels; neo-angiogenesis. This picture is characteristic of moyamoya syndrome secondary to intracranial vasculitis. The angiography findings are shown in Fig. 2.

Laboratory investigations revealed normal rheumatoid factor $(12 \mathrm{IU} / \mathrm{ml})$, normal anti-CCP antibodies (4.3 unit $/ \mathrm{ml})$, elevated C-reactive protein $(14 \mathrm{mg} / \mathrm{L})$, elevated erythrocyte sedimentation rate $(81 \mathrm{~mm} / \mathrm{h})$, normal hemoglobin level (12.9 gm/dL), normal white blood cells count $\left(6120 / \mathrm{mm}^{3}\right)$, normal platelets count $(280,000$ / $\left.\mathrm{mm}^{3}\right)$, normal prothrombin time (10.5 s) and normal partial thromboplastin time (32.4 s). A transthoracic echocardiogram was unremarkable.

The diagnosis of RA was made several years ago and the patient was on oral prednisolone $5 \mathrm{mg}$ per day and leflunomide $20 \mathrm{mg}$ per day but she was non-compliant. Rheumatologic consultation was done and hydroxychloroquine $400 \mathrm{mg}$ per day was added to her treatment. The patient was discharged after 1 week of hospital admission with disappearance of the headache and partial recovery of her aphasia symptoms as she could understand simple commands with appropriate response.

\section{Discussion}

Collagen diseases are often complicated by cerebrovascular disorders; however, these rarely occur in RA [4]. The rate of occurrence of cerebral vasculitis in patients with RA is $1-8 \%$ [5].

The most frequent neurological complications with RA are atlantoaxial subluxation, polymyositis, 


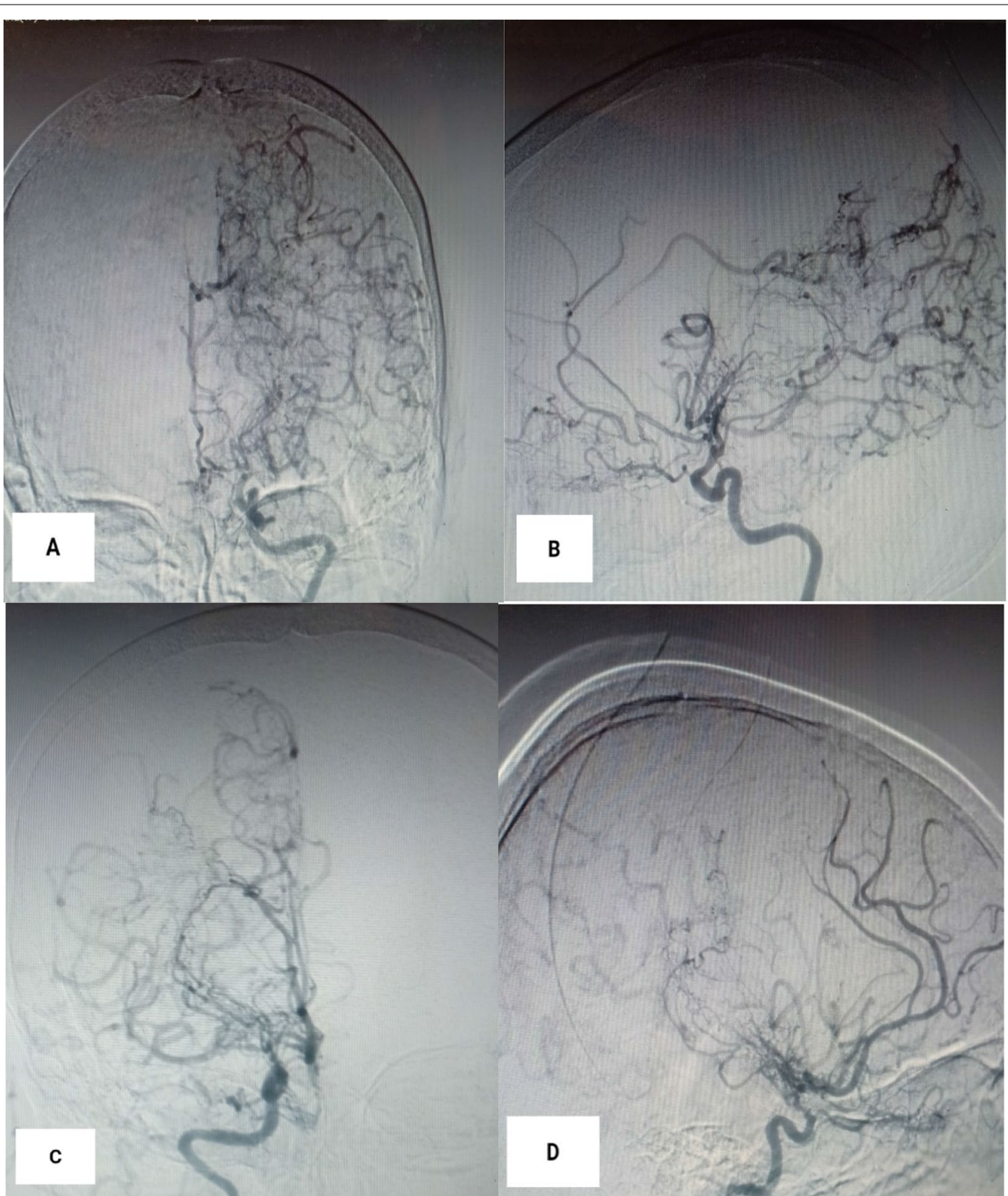

Fig. 2 A Left internal carotid artery (ICA) angiogram anterior view showing occlusion of its distal part of with neo-angiogenesis. B Left ICA angiogram lateral view. C Right ICA angiogram anterior view showing occlusion of its distal part with neo-angiogenesis. D Right ICA angiogram lateral view

mononeuritis multiplex, peripheral neuropathy, rheumatoid nodules within the central or peripheral nervous system, and RV [6].They were initially described in seropositive patients with long history of active disease, with subcutaneous nodules and extra-articular manifestations [7].

Our case was seronegative and had a long history of active RA, but no previous extra-articular manifestations.

The neurological manifestations of cerebral RV include hemiparesis, focal onset epilepsy, cranial nerve palsy, visual field impairment, altered consciousness, and cognitive impairment $[8,9]$.

The neurological manifestations in our case were headache and receptive aphasia due to the occurrence of intracerebral hemorrhage in absence of other risk factors for hemorrhage.

Two previous studies evaluated various autoimmune diseases and the risk of $\mathrm{ICH}$. Both studies found that the danger of ICH is higher in patients with RA, with the highest risk occurring during the first year of diagnosis 
[10]. In a meta-analysis of 23 studies, it was found that patients with RA have a risk of hemorrhagic stroke 1.68 times higher than that in normal individuals [11].

Moyamoya disease is a rare neurovascular disorder of unknown etiology characterized by progressive stenosis or occlusion of the supraclinoid portion of both internal carotid arteries and development of collateral vessels. The classical presentations of the disease in children are transient ischemic attacks and seizures, whereas in adults, cerebral ischemia and cerebral hemorrhage are the commonest manifestations [12].

Moyamoya syndrome or secondary moyamoya disease has similar angiographic findings as primary moyamoya disease. Moyamoya syndrome can be seen in patients with cerebral vasculitis, fibromuscular dysplasia, sickle cell disease, and advanced intracranial atherosclerosis. The incidence of moyamoya syndrome in patients with RA is unclear due to lack of sufficient data [13].

Our case showed moyamoya syndrome angiographic pattern with DSA, denting chronic intracranial vasculitis. Also, intracerebral hemorrhage was the presenting manifestation as reported in the literature.

\section{Conclusions}

Central nervous system involvement in rheumatic arthritis may be associated with high morbidity and can be a life-threatening condition. In rheumatoid disease, early diagnosis, proper treatment, good adherence to treatment plan and regular follow-up visits are essential in preventing serious complications.

\section{Abbreviations}

Anti-CCP: Anti-cyclic citrullinated peptide; CT brain: Computed tomography of the brain; DSA: Digital subtraction angiography; ICH: Intracerebral hemorrhage; ICU: Intensive care unit; RA: Rheumatoid arthritis; RV: Rheumatoid vasculitis.

\section{Acknowledgements \\ Not applicable.}

\section{Authors' contributions}

$\mathrm{AH}$ : design and conceptualized the study. AE, SS, AS: conception of the work and drafting the manuscript. $\mathrm{SH}, \mathrm{RY}, \mathrm{MH}$ : acquisition and analysis of data. All authors have read and approved the final manuscript.

\section{Funding}

No funds were received to fulfill this work.

\section{Availability of data and materials}

The corresponding author takes full responsibility for the data, has full access to all of the data, and has the right to publish any and all data separate and apart from any sponsor.

\section{Declarations}

\section{Ethics approval and consent to participate}

All procedures performed in the study were in accordance with the ethical standards of the Faculty of Medicine, Ain Shams University Research and
Ethical Committee, and with the 1964 Helsinki declaration and its later amendments or comparable ethical standards. We obtained approval from the research ethics committee on February 2021.

\section{Consent for publication}

Written informed consent was obtained from the participant for publication of this case and accompanying images.

\section{Competing interests}

The authors declare that they do not have any competing interests.

\section{Author details}

${ }^{1}$ Neurology Department, Faculty of Medicine, Ain Shams University, Cairo, Egypt. ${ }^{2}$ Internal Medicine Department, Faculty of Medicine, Ain Shams University, Cairo, Egypt.

Received: 30 June 2021 Accepted: 7 October 2021

Published online: 16 October 2021

References

1. Del Rincón I, Williams K, Stern MP, Freeman GL, O'Leary DH, et al. Association between carotid atherosclerosis and markers of inflammation in rheumatoid arthritis patients and healthy subjects. Arthritis Rheum. 2003;48(7):1833-40.

2. Zöller B, Li X, Sundquist J, Sundquist K. Risk of subsequent ischemic and hemorrhagic stroke in patients hospitalized for immune-mediated diseases: a nationwide follow-up study from Sweden. BMC Neurol. 2012;12:41

3. Kousa O, Awad DH, Hydoub YM, Awawdeh R, Andukuriet V. Intracerebral hemorrhage in a patient with untreated rheumatoid arthritis: case report and literature review. Cureus. 2019;11(7):e5175.

4. Ando Y, Kai S, Uyama E, Lyonaga K, Hashimoto Y, et al. Involvement of the central nervous system in rheumatoid arthritis: its clinical manifestations and analysis by magnetic resonance imaging. Intern Med. 1995:34(3):188-91.

5. Watts RA, Mooney J, Lane SE, Scott DG. Rheumatoid vasculitis: becoming extinct? Rheumatology (Oxford). 2004;43(7):920-3.

6. Sofat N, Malik O, Higgens CS. Neurological involvement in patients with rheumatic disease. QJM. 2006;99(2):69-79.

7. Pons NC, Montalà N, Valverde J, Brell M, Ferrer I, et al. Isolated cerebral vasculitis associated with rheumatoid arthritis. Jt Bone Spine. 2010;77(4):361-3.

8. Mrabet D, Meddeb N, Ajlani H, Sahli H, Sellami S. Cerebral vasculitis in a patient with rheumatoid arthritis. Jt Bone Spine. 2007;74(2):201-4.

9. Akrout R, Bendjemaa S, Fourati H, Ezzeddine M, Hachicha I, et al. Cerebral rheumatoid vasculitis: a case report. J Med Case Rep. 2012;6:302.

10. Ramagopalan SV, Pakpoor J, Seminog O, Goldacre R, Graham L, et al. Risk of subarachnoid haemorrhage in people admitted to hospital with selected immune-mediated diseases: record-linkage studies. BMC Neurol. 2013;13:176.

11. Wiseman SJ, Ralston SH, Wardlaw JM. Cerebrovascular disease in rheumatic diseases: a systematic review and meta-analysis. Stroke. 2016:47(4):943-50.

12. Suzuki J, Takaku A. Cerebrovascular 'moyamoya' disease. Disease showing abnormal net-like vessels in base of brain. Arch Neurol. 1969;20(3):288-99.

13. Scott RM, Smith ER. Moyamoya disease and moyamoya syndrome. N Engl J Med. 2009;360(12):1226-37.

\section{Publisher's Note}

Springer Nature remains neutral with regard to jurisdictional claims in published maps and institutional affiliations. 